\title{
A new proof of the non-tameness of the Nagata automorphism from the point of view of the Sarkisov program
}

\author{
Takashi Kishimoto \\ Dedicated to Professor Masayoshi Miyanishi on the occasion of his 65th birthday.
}

\begin{abstract}
The Nagata automorphism is a kind of complicated automorphism on the affine 3 -space $\mathbb{C}^{3}$. For a long time, it remained unknown whether or not the Nagata automorphism is tame until Shestakov and Umirbaev at last proved that it is not tame in 2004, by purely algebraic methods (e.g. Poisson algebra). In this paper, we consider a certain necessary condition for a given automorphism on $\mathbb{C}^{3}$ to be tame from the point of view of the Sarkisov program established by Corti. Furthermore, by using it, we shall give a new algebro-geometric proof of the non-tameness of the Nagata automorphism.
\end{abstract}

\section{Introduction}

Throughout the present paper we work over the field of complex numbers $\mathbb{C}$. In affine algebraic geometry, it is important to understand the structure of the group of automorphisms $G_{n}:=$ $\operatorname{Aut}_{\mathbb{C}}\left(\mathbb{C}\left[x_{1}, \ldots, x_{n}\right]\right)$ on polynomial rings in $n$ variables over $\mathbb{C}$. An automorphism $\tau$ on $\mathbb{C}\left[x_{1}, \ldots, x_{n}\right]$ is called affine (respectively de Jonquière) if $\tau\left(x_{i}\right)=\sum_{j=1}^{n} a_{i j} x_{j}+b_{i}$ with $a_{i j}, b_{i} \in \mathbb{C}$ and $\operatorname{det}\left(a_{i j}\right) \neq 0$ (respectively $\tau\left(x_{i}\right)=a_{i} x_{i}+f_{i}\left(x_{i+1}, \ldots, x_{n}\right)$ with $a_{i} \neq 0, f_{i} \in \mathbb{C}\left[x_{i+1}, \ldots, x_{n}\right](1 \leqslant i<n)$ and $f_{n} \in \mathbb{C}$ ). We shall introduce three subgroups of $G_{n}$. Let $A_{n}$ (respectively $J_{n}$ ) be the subgroup of $G_{n}$ consisting of all affine transformations (respectively de Jonquière transformations). We denote by $T_{n}$ the subgroup of $G_{n}$ generated by $A_{n}$ and $J_{n}$. An automorphism $\tau$ is said to be tame if it is contained in $T_{n}$. For the case $n=2$, it is classically known that all automorphisms on $\mathbb{C}\left[x_{1}, x_{2}\right]$ are tame, i.e. $G_{2}=T_{2}$ (cf. [Nag72, AM75, Miy78]). On the other hand, for the higher-dimensional case $n \geqslant 3$, we know very little concerning the structure of $G_{n}$. In what follows, we shall especially pay attention to the case $n=3$, and write $\mathbb{C}[x, y, z]$ instead of the notation $\mathbb{C}\left[x_{1}, x_{2}, x_{3}\right]$. In order to indicate the complexity of the group $G_{3}$, let us consider the following famous automorphism, the so-called Nagata automorphism (cf. [Nag78, p. 16]):

$$
\sigma:\left\{\begin{array}{l}
x \mapsto x-2 y\left(x z+y^{2}\right)-z\left(x z+y^{2}\right)^{2}, \\
y \mapsto y+z\left(x z+y^{2}\right), \\
z \mapsto z
\end{array}\right.
$$

Although the Nagata automorphism $\sigma$ seems to be simple, for a long time it remained unknown whether or not $\sigma$ is tame. Although the structure on $G_{3}$ itself cannot be understood in detail at the present time, Shestakov and Umirbaev developed a technique to decide the tameness of a given

Received 25 January 2007, accepted in final form 24 August 2007.

2000 Mathematics Subject Classification 14R10 (primary), 14E07 (secondary).

Keywords: Nagata automorphism, Sarkisov program, elementary links.

This journal is (C) Foundation Compositio Mathematica 2008. 


\section{T. Кіsнiмото}

automorphism on $\mathbb{C}[x, y, z]$ by purely algebraic methods (cf. [SU04a, SU04b]). As a consequence, they proved at last that $\sigma$ is, indeed, not tame, i.e. $\sigma \notin T_{3}$. However, as mentioned just above, since their method is purely algebraic based on the treatment of Poisson algebra, it is complicated and lacks the intrinsic geometry hiding in the Nagata automorphism $\sigma$. One of the main purposes in this paper is to give an alternative proof of non-tameness on $\sigma$ from the point of view of birational geometry, more precisely, the Sarkisov program (cf. [Cor95, Cor00, Mat01, Kis05]). Roughly speaking, the Sarkisov program gives us a useful tool for the factorization of a given birational map between three-dimensional Mori fiber spaces into certain kinds of simple birational maps, so-called elementary links. But, in general, it is difficult to perform explicit factorizations into elementary links. This difficulty mainly seems to result from the lack of classification of three-dimensional terminal divisorial contractions. (Nevertheless, there exists something remarkable concerning the classification of three-dimensional terminal divisorial contractions due to Kawamata, Kawakita and Tziolas et al.; see [Kaw96, Ka01, Ka02, Ka05, Tzi03].) We shall make use of an algorithm of the Sarkisov program (see $\S 2$ ) for the investigation of the Cremona transformation $\Phi_{\theta}: \mathbb{P}^{3}-->\mathbb{P}^{3}$ induced by an automorphism $\theta \in G_{3}$ on $\mathbb{C}^{3}$. The explicit Sarkisov factorization of $\Phi_{\theta}$ itself is usually difficult to perform. However, it is possible, in theory, to determine the maximal center (cf. Definition 2.2) of the first elementary link appearing in the Sarkisov factorization of $\Phi_{\theta}$ once $\theta$ is given concretely. As far as we are concerned with the (non-)tameness of a given $\theta$, the investigation of a maximal center of the first elementary link gives us useful information. Namely, we prove the following theorem.

Theorem 1.1. Let $\theta$ be a tame automorphism on the affine 3 -space $\mathbb{C}^{3}$, and $\Phi_{\theta}$ the Cremona transformation on $\mathbb{P}^{3}$ induced by $\theta$ in a natural way. Then, for any Sarkisov factorization of $\Phi_{\theta}$, say

$$
\Phi_{\theta}=\chi_{s}^{\prime} \circ \cdots \circ \chi_{1}^{\prime},
$$

the maximal center of the maximal divisorial blow-up (Definition 2.2) appearing in the first elementary link $\chi_{1}^{\prime}$ is either a point or a line on the hyperplane at infinity.

Once we have obtained Theorem 1.1, we can prove the following result.

TheOREM 1.2 (cf. [SU04a, SU04b]). The Nagata automorphism is not tame.

In fact, the Nagata automorphism $\sigma$ is naturally extended to the Cremona transformation $\Phi_{\sigma}$ : $\mathbb{P}^{3} \cdots \rightarrow \mathbb{P}^{3}$ as in the following fashion:

$$
\Phi_{\sigma}:\left\{\begin{array}{l}
x \mapsto x t^{4}-2 y\left(x z+y^{2}\right) t^{2}-z\left(x z+y^{2}\right)^{2}, \\
y \mapsto\left(y t^{2}+z\left(x z+y^{2}\right)\right) t^{2} \\
z \mapsto z t^{4} \\
t \mapsto t^{5}
\end{array}\right.
$$

where the hyperplane at infinity $H_{\infty}$ with respect to the canonical open immersion $\mathbb{C}^{3}(x, y, z) \hookrightarrow \mathbb{P}^{3}$ is defined by $t=0$. As for this Cremona transformation, fortunately, in our previous paper [Kis05], we succeeded in the explicit factorization of $\Phi_{\sigma}$ into eight elementary links by making use of the Sarkisov program. According to this, we see that the first elementary link starts with a blow-up along a smooth conic on $H_{\infty}$ to deduce that $\sigma$ is not tame by Theorem 1.1. However, in consideration of Theorem 1.1, it is useful to give a proof of the non-tameness of $\sigma$ that depends only on the investigation of a maximal center of the first link, for further applications. That is why we shall give a new proof from this viewpoint in $\S 4$.

We shall state the scheme of this paper. In $\S 2$, we recall the definitions of the Sarkisov program and elementary links. In particular, Sarkisov degree and maximal center are indispensable in order to explain the mechanism of the Sarkisov program. But, since we are able to find accessible explanations 
in many articles (cf. [Cor95, Cor00, Mat01]), we only summarize in brief. In $\S 3$, we give a proof of Theorem 1.1. In $\S 4$, we give a proof of Theorem 1.2, that is, a new proof on the non-tameness of the Nagata automorphism $\sigma$, which relies only on the investigation of a maximal center of the first elementary link appearing in the Sarkisov factorization of $\Phi_{\sigma}$, as an application of Theorem 1.1. In fact, we can see that a maximal center must be a smooth conic on the hyperplane at infinity. Here, for the exclusion of other subvarieties as maximal centers, we make use of the technique due to Corti in $[$ Cor00, §3]. We think that the new proof lets us recognize the importance and utility of the Sarkisov program for the further investigation of automorphisms on $\mathbb{C}[x, y, z]$ from an algebro-geometric viewpoint.

\section{Notation, convention and definition}

In this paper, we use the following:

(i) $K_{V}$, the canonical divisor of a variety $V$;

(ii) mult $_{W} D$, the multiplicity of a scheme $D$ on $V$ along a subvariety $W \subset D$;

(iii) $\sim$, the linear equivalence;

(iv) $\equiv$, the numerical equivalence;

(v) $a\left(\nu ; K_{V}+\alpha \mathcal{H}_{V}\right)$, the discrepancy of a discrete valuation $\nu$ of the function field $\mathbb{C}(V)$ of $V$ with respect to $K_{V}+\alpha \mathcal{H}_{V}$;

(vi) $\operatorname{Center}_{V}(\nu)$, the center on $V$ of a discrete valuation $\nu$ of $\mathbb{C}(V)$.

\section{Summary of the Sarkisov program}

In this section, we shall summarize the Sarkisov program. Since accessible explanations concerning the Sarkisov program can be found in many articles [Cor95, Cor00, Mat01, KSC04], we do not repeat these here in detail, and we shall only prepare some indispensable terminology and sketch the mechanism to apply the Sarkisov program in the subsequent sections of this paper. In what follows in this section, we use 'Mfs' instead of a three-dimensional Mori fiber space for short. The Sarkisov program gives us a useful algorithm to factorize a given birational map between two Mfs into some kind of simple birational maps between Mfs, so-called elementary links (cf. [Cor95, Cor00]). The elementary links are divided into four types (types I, II, III and IV) as in the following diagrams.

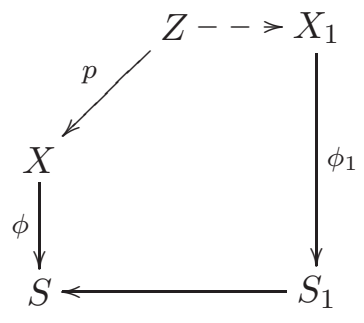

Type I

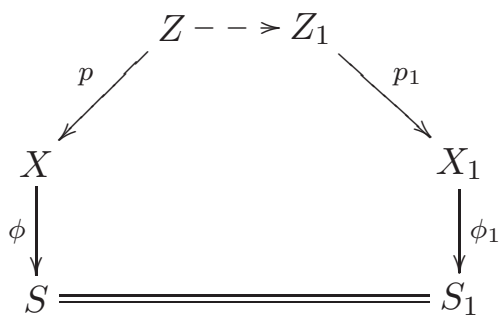

Type II 


\section{T. Кіsнiмото}

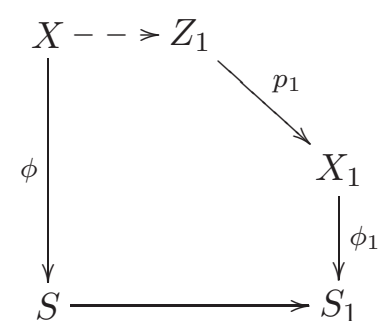

Type III

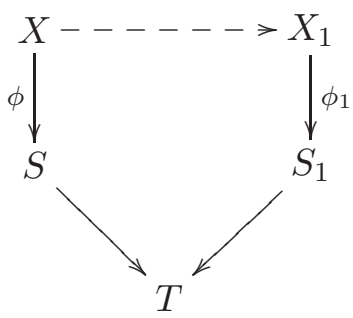

Type IV

Here $p: Z \rightarrow X$ and $p_{1}: Z_{1} \rightarrow X_{1}$ are the divisorial contractions (or the maximal divisorial blowup; see Definition 2.2 below) and each of the birational maps denoted by horizontal dotted arrows, which may be an identity, is a composite of log flips (i.e. inverse flips, flops and flips), $\phi: X \rightarrow S$, $\phi_{1}: X_{1} \rightarrow S_{1}$ are Mfs and $\varrho(X / S)=\varrho\left(X_{1} / S_{1}\right)=\varrho(S / T)=\varrho\left(S_{1} / T\right)=1$.

We shall recall the definition of the Sarkisov degree. Let $\varphi: X / S-->X^{\prime} / S^{\prime}$ be a given birational map between Mfs $\phi: X \rightarrow S$ and $\phi^{\prime}: X^{\prime} \rightarrow S^{\prime}$.

Definition 2.1 (Sarkisov degree). Let $\mathcal{H}^{\prime}=\left|-\mu^{\prime} K_{X^{\prime}}+\phi^{\prime *}\left(A^{\prime}\right)\right|$ be a very ample complete linear system on $X^{\prime}$, where $\mu^{\prime}$ is a positive integer and $A^{\prime}$ is a sufficiently ample Cartier divisor on $S^{\prime}$. This choice of $\mathcal{H}^{\prime}$ is made once and for all at the beginning of the factorization process and $\mathcal{H}^{\prime}$ remains unchanged throughout. Let $\mathcal{H}:=\varphi_{*}^{-1}\left(\mathcal{H}^{\prime}\right)$ be the proper transform of $\mathcal{H}^{\prime}$ by $\varphi$. Since $\phi: X \rightarrow S$ is Mfs, $\mathcal{H}$ is written uniquely as $\mathcal{H} \equiv-\mu K_{X}+\phi^{*}(A)$, where $\mu$ is a positive rational number and $A$ is a $\mathbb{Q}$-Cartier, $\mathbb{Q}$-divisor on $S$. This $\mu$ is called the quasi-effective threshold of the pair $(X, \mathcal{H})$. Let $c:=\sup \left\{\lambda \in \mathbb{Q}: K_{X}+\lambda \mathcal{H}\right.$ is canonical $\}$, which is said to be the canonical threshold of $(X, \mathcal{H})$. (In the case Bs $\mathcal{H}=\emptyset$ we put $c=+\infty$.) Finally, let $e$ be the number of discrete valuations $\{E\}$ of $\mathbb{C}(X)$ which are exceptional over $X$ and have discrepancy $a\left(E ; K_{X}+c \mathcal{H}\right)=0$. (Note that $e \geqslant 0$ is finite.) The triplet $(\mu, c, e)$ is called the Sarkisov degree of $(X, \mathcal{H})$.

We shall define the lexicographical order on the set of Sarkisov degrees as follows. When two birational maps $X / S-\stackrel{\varphi}{\rightarrow} X^{\prime} / S^{\prime} \leftarrow--X_{1} / S_{1}$ between Mfs are given, we have two Sarkisov degrees $(\mu, c, e)$ and $\left(\mu_{1}, c_{1}, e_{1}\right)$ of the pairs $(X, \mathcal{H})$ and $\left(X_{1}, \mathcal{H}_{1}\right)$ as defined just above, where $\mathcal{H}$ (respectively $\mathcal{H}_{1}$ ) is the proper transform of $\mathcal{H}^{\prime}$ by $\varphi$ (respectively $\left.\varphi_{1}\right)$. We write $(\mu, c, e)>\left(\mu_{1}, c_{1}, e_{1}\right)$ if one of the following is satisfied:

(i) $\mu>\mu_{1}$, or

(ii) $\mu=\mu_{1}$ and $c<c_{1}$ (not miswriting!!), or

(iii) $\mu=\mu_{1}, c=c_{1}$ and $e>e_{1}$.

The essential idea in the Sarkisov program lies in untwisting the original birational map $\varphi$ by suitable elementary links to decrease the Sarkisov degree and to use the Noether-Fano-Iskovskih inequality (cf. [Cor95, Cor00, Mat01]). We shall sketch the mechanism of the Sarkisov program to factorize $\varphi: X / S-\rightarrow X^{\prime} / S^{\prime}$. The reader should refer to [Cor95, Cor00, Mat01] for a more detailed explanation. Roughly speaking, the four kinds of elementary links (types I-IV) are divided into two groups, types I and II, and types III and IV, according as $K_{X}+(1 / \mu) \mathcal{H}$ is canonical or not.

If $K_{X}+(1 / \mu) \mathcal{H}$ is canonical and nef, then $\varphi$ is an isomorphism as Mfs by virtue of the NoetherFano-Iskovskih inequality (cf. [Cor95, Cor00]) and there is nothing to do. Otherwise, we need to consider whether $K_{X}+(1 / \mu) \mathcal{H}$ is canonical or not, separately.

In the case that $K_{X}+(1 / \mu) \mathcal{H}$ is canonical but not nef, we can find a $\left(K_{X}+(1 / \mu) \mathcal{H}\right)$-negative extremal ray, say $\mathbb{P}$, by the cone theorem (cf. [KMM87]), and consider the extremal face $\mathbb{F}$ spanned 
by $\mathbb{P}$ and $\mathbb{R}$, where $\mathbb{R}$ is an extremal ray giving rise to Mfs $\phi: X \rightarrow S$. Let $\operatorname{cont}_{\mathbb{F}}: X \rightarrow T$ be the contraction of $\mathbb{F}$. Note that since $\mathbb{R} \subset \mathbb{F}$, the contraction cont $_{\mathbb{F}}$ factors through $\phi=\operatorname{cont}_{\mathbb{R}}: X \rightarrow S$. We run $\left(K_{X}+(1 / \mu) \mathcal{H}\right)$-MMP over $T$. Then we reach at the end either a log minimal model or a $\log$ Mfs (with respect to $K_{X}+(1 / \mu) \mathcal{H}$ over $T$ ). Thus we obtain a link of type III or IV according as the last birational contraction in this $\left(K_{X}+(1 / \mu) \mathcal{H}\right)$-MMP over $T$ is divisorial or not.

On the other hand, if $K_{X}+(1 / \mu) \mathcal{H}$ is not canonical, i.e. $c<1 / \mu$, then we need to take a suitable birational modification according to the singularities of a general member of $\mathcal{H}$ as follows.

Definition 2.2. We call $p: Z \rightarrow X$ a maximal divisorial blow-up with respect to $K_{X}+c \mathcal{H}$ if $p: Z \rightarrow X$ is a projective birational morphism from $Z$ with only $\mathbb{Q}$-factorial, terminal singularities such that:

(i) $\varrho(Z / X)=1$;

(ii) the exceptional locus of $p$ is a prime divisor; and

(iii) $p$ is $\left(K_{X}+c \mathcal{H}\right)$-crepant, i.e. $K_{Z}+c \mathcal{H}_{Z} \equiv p^{*}\left(K_{X}+c \mathcal{H}\right)$, where $\mathcal{H}_{Z}$ is the proper transform of $\mathcal{H}$ on $Z$.

A discrete valuation $E$ of $\mathbb{C}(X)$ is said to be a (strong) maximal singularity of $\mathcal{H}$ if $E$ is the exceptional divisor of a suitable maximal divisorial blow-up. A maximal center is the center $\operatorname{Center}_{X}(E)$ on $X$ of a maximal singularity $E$.

Remark 2.1. If $K_{X}+(1 / \mu) \mathcal{H}$ is not canonical, then $\mathcal{H}$ has a base locus Bs $\mathcal{H} \neq \emptyset$ and, furthermore, a maximal center of $\mathcal{H}$ is contained in $\mathrm{Bs} \mathcal{H}$.

It is known that a maximal divisorial blow-up with respect to $K_{X}+c \mathcal{H}$ always exists (cf. [Mat01, Proposition 13-1-8]). After taking a maximal divisorial blow-up $p: Z \rightarrow X$ with respect to $K_{X}+c \mathcal{H}$, we run $\left(K_{Z}+c \mathcal{H}_{Z}\right)$-MMP over $S$. Then we necessarily reach a $\log$ Mfs $\phi_{1}: X_{1} \rightarrow S_{1}$. (Note that the case where we reach a log minimal model over $S$ does not occur.) According to whether the last birational contraction in the $\left(K_{Z}+c \mathcal{H}_{Z}\right)$-MMP over $S$ is divisorial or not, we obtain an elementary link of type II or type I.

Thus we can construct an elementary link $\varphi_{1}: X / S-->X_{1} / S_{1}$ of type I-IV. It can be verified that the Sarkisov degree of $\left(X_{1}, \mathcal{H}_{1}\right)$, say $\left(\mu_{1}, c_{1}, e_{1}\right)$, decreases strictly compared with that of $(X, \mathcal{H})$. Therefore we have a new birational map $\varphi \circ \varphi_{1}^{-1}: X_{1} / S_{1}->X^{\prime} / S^{\prime}$ between Mfs with strictly smaller Sarkisov degree $\left(\mu_{1}, c_{1}, e_{1}\right)$. We then ask again whether or not $K_{X_{1}}+\left(1 / \mu_{1}\right) \mathcal{H}_{1}$ is canonical and nef. If the answer is yes, then $\varphi \circ \varphi_{1}^{-1}: X_{1} / S_{1}-->X^{\prime} / S^{\prime}$ is an isomorphism by the NoetherFano-Iskovskih inequality to see $\varphi=\varphi_{1}$. On the other hand, if the answer is no, we repeat the above mentioned process with $\varphi$ replaced by $\varphi \circ \varphi_{1}^{-1}$. Each time we untwist, the Sarkisov degree should strictly drop. Finally, it is known that this process has to come to an end after finitely many repetitions to obtain the expression of $\varphi$ as a composite of elementary links, which we shall call the Sarkisov factorization of $\varphi$ directed by $\mathcal{H}$.

\section{Maximal centers of Cremona transformations on $\mathbb{P}^{3}$ induced by tame automorphisms on $\mathbb{C}^{3}$}

As explained in $\S 2$, the Sarkisov program gives us a useful algorithm to factorize a given birational map among Mfs into elementary links. The elementary links of types I and II start with suitable maximal divisorial blow-ups along maximal centers (Definition 2.2). In many applications of the Sarkisov program to explicit birational geometry, it is essential to investigate maximal centers. For instance, this plays a substantial role in showing that there exist very few Mori fiber spaces which are birational to some kinds of $\mathbb{Q}$-Fano 3-folds, e.g. quartic 3-folds admitting a certain kind of singularity (cf. [Cor00, CPR00, CM04, Mel04]). In this section, we shall consider especially a 


\section{T. Кіsнiмото}

Cremona transformation $\Phi_{\theta}$ on $\mathbb{P}^{3}$, which is induced by a tame automorphism $\theta$ on the inside affine 3 -space $\mathbb{C}^{3}$, and investigate a maximal center of the first elementary link when applying the Sarkisov program for $\Phi_{\theta}$. As seen in Theorem 1.1, for any Sarkisov factorization of $\Phi_{\theta}$, the maximal center of the first elementary link is either a point or a line on the hyperplane at infinity provided that $\theta$ is tame. First of all, we shall restrict the possibility of maximal centers of the first elementary link without the tameness assumption.

Proposition 3.1. Let $\theta \in G_{3}$ be an automorphism on the affine 3 -space $\mathbb{C}^{3}$ (which is not necessarily tame), and let $\Phi_{\theta}: \mathbb{P}^{3}--\rightarrow \mathbb{P}^{3}$ be the Cremona transformation induced by $\theta$ in a natural way. Then the maximal center of the first elementary link of the Sarkisov factorization of $\Phi_{\theta}$ is either a point, a line or a smooth conic on the hyperplane at infinity.

Proof. We put $f(x, y, z):=\theta(x), g(x, y, z):=\theta(y)$ and $h(x, y, z):=\theta(z)$, and let $F(x, y, z, t)$, $G(x, y, z, t)$ and $H(x, y, z, t)$ be the corresponding homogeneous polynomials of $f, g$ and $h$, respectively. Put $d:=\max \{\operatorname{deg}(f), \operatorname{deg}(g), \operatorname{deg}(h)\}$, and we may assume that $d=\operatorname{deg}(f)$. Then, by the mechanism of the Sarkisov program (see [Cor95]; see also $\S 2$, Remark 2.1), it is easy to see that the maximal center of the first elementary link $\chi_{1}$, say $C_{0}$, is contained in the plane curve of degree $d$ :

$$
C:=(F(x, y, z, 0)=0) \subset H_{\infty} \cong \mathbb{P}_{[x: y: z]}^{2},
$$

where $H_{\infty}$ is the hyperplane at infinity with respect to the canonical open immersion $\mathbb{C}^{3} \hookrightarrow \mathbb{P}^{3}$. Note that since $C$ is a boundary of the affine plane $(f=0) \cong \mathbb{C}^{2}$, each irreducible component of $C$ is rational and $C$ does not contain a circular chain (cf. [Miy01]). Provided that the maximal center $C_{0}$ is not a point on $H_{\infty}$, it coincides with a suitable (rational) curve contained in $C$. Since $\mathbb{P}^{3}$ is smooth, $\chi_{1}$ starts with just the blow-up along the plane curve $C_{0}$, say $p: Z \rightarrow \mathbb{P}^{3}$ (cf. [Mor82]). If $\operatorname{deg} C_{0} \geqslant 3$, then $C_{0}$ has a singular point, so that the resulting 3 -fold $Z$ has a singular locus of dimension one. This implies that $Z$ cannot be terminal, which is a contradiction to the mechanism of the Sarkisov program (see $\S 2$ ). Hence it follows that $C_{0}$ is either a line or a (smooth) conic.

In what follows, we assume that $\theta$ is a tame automorphism on $\mathbb{C}^{3}$. Since $\theta$ is tame, it is written as in the following fashion:

$$
\theta=\tau_{N+1} \circ \sigma_{N} \circ \tau_{N} \circ \cdots \circ \sigma_{1} \circ \tau_{1}
$$

where $\tau_{j} \in A_{3}$ (respectively $\sigma_{i} \in J_{3} \backslash A_{3}$ ) is an affine transformation (possibly an identity) (respectively de Jonquière transformation but is not affine) on $\mathbb{C}^{3}$.

LEMMA 3.1. We have

$$
\Phi_{\theta}=\Phi_{\tau_{N+1}} \circ \Phi_{\sigma_{N}} \circ \Phi_{\tau_{N}} \circ \cdots \circ \Phi_{\sigma_{1}} \circ \Phi_{\tau_{1}},
$$

where $\Phi_{\tau_{j}}$ (respectively $\Phi_{\sigma_{i}}$ ) is the Cremona transformation on $\mathbb{P}^{3}$ arising from $\tau_{j}$ (respectively $\sigma_{i}$ ) in a natural way.

Proof. It is clear to see the assertion.

Since the Sarkisov program gives us an algorithm of factorizations up to automorphisms on $\mathbb{P}^{3}$, we may assume that $\Phi_{\tau_{1}}=\Phi_{\tau_{N+1}}=$ id in (**) to obtain our result (Theorem 1.1). We factorize each Cremona transformation $\Phi_{\sigma_{i}}$ induced from $\sigma_{i} \in J_{3} \backslash A_{3}$ into elementary links according to the Sarkisov program directed by a suitable mobile linear system (cf. $\S 2$, and [Cor95]), say

$$
\Phi_{\sigma_{i}}=\chi_{r_{i}}^{(i)} \circ \cdots \circ \chi_{1}^{(i)} \quad \text { (up to linear transformations on } \mathbb{P}^{3} \text { ), }
$$

where $\chi_{l}^{(i)}$ is an elementary link for $1 \leqslant l \leqslant r_{i}$. Thus, by substituting, we have the factorization of $\Phi_{\theta}$ :

$$
\Phi_{\theta}=\left(\chi_{r_{N}}^{(N)} \circ \cdots \circ \chi_{1}^{(N)}\right) \circ \Phi_{\tau_{N}} \circ \cdots \circ \Phi_{\tau_{2}} \circ\left(\chi_{r_{1}}^{(1)} \circ \cdots \circ \chi_{1}^{(1)}\right)
$$

into elementary links up to linear transformations on $\mathbb{P}^{3}$. 


\section{Proof of NON-TAMENESS OF THE NAGATA AUtOMORPhisM}

Although $(* * *)$ does not necessarily come from Sarkisov factorization, it gives us important information by combining with the result due to Freudenburg [Fre95, Theorem 1.1.1]. From now on for the time being, we shall state the result in [Fre95] in the form which is enough for our purpose. (In fact, Freudenburg [Fre95] has proved more general results concerning automorphisms on projective 3 -folds of the forms $\mathcal{F}_{n}, \mathcal{F}_{(m, n)}$ and $\mathcal{F}_{(m, n)}^{u}$; see Remark 3.2 for the definition of these 3-folds.)

Theorem 3.1 (cf. [Fre95, Theorem 1.1.1]). Let $\alpha$ be an automorphism of de Jonquière type on the affine 3 -space $\mathbb{C}^{3}$. Then there exists an algebraic 3-fold $V$ of the form either $V \cong \mathcal{F}_{n}, \mathcal{F}_{(m, n)}$ or $\mathcal{F}_{(m, n)}^{u}$ for suitable non-negative integers $m, n, u \in \mathbb{Z}$ (see [Fre95] for the definitions of $\mathcal{F}_{n}, \mathcal{F}_{(m, n)}$ and $\mathcal{F}_{(m, n)}^{u}$; see also Remark 3.2), which contains $\mathbb{C}^{3}$ as a Zariski open subset, such that $\alpha$ is extended to an automorphism on $V$.

Remark 3.1. Algebraic 3-folds $V \cong \mathcal{F}_{n}, \mathcal{F}_{(m, n)}$ and $\mathcal{F}_{(m, n)}^{u}$ as in Theorem 3.1 have the structure of a $\mathbb{P}^{1}$-bundle over $\mathbb{P}^{2}$, a $\mathbb{P}^{2}$-bundle over $\mathbb{P}^{1}$ and an $\mathbb{F}_{u}$-bundle over $\mathbb{P}^{1}$, respectively (cf. Remark 3.2 ). There exists a birational map $\chi: \mathbb{P}^{3}-->V$, which does not have any influence on the inside $\mathbb{C}^{3}$, such that it fits into the following commutative diagram.

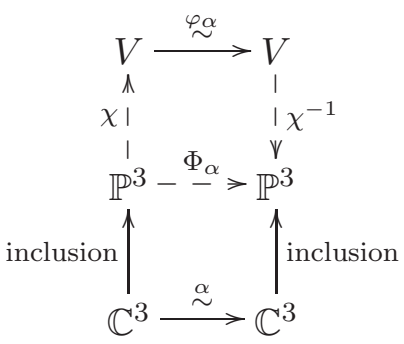

Here $\Phi_{\alpha}: \mathbb{P}^{3}--\rightarrow \mathbb{P}^{3}$ is, as usual, the Cremona transformation arising from $\alpha \in J_{3}$ in a natural fashion, and $\varphi_{\alpha}: V \stackrel{\sim}{\longrightarrow} V$ is an automorphism whose existence is guaranteed by Theorem 3.1. More precisely, we can describe the procedure $\chi: \mathbb{P}^{3}-\rightarrow V$ explicitly as a composite of so-called elementary transformations in terms of [Fre95]. Via these elementary transformations, several $\mathcal{F}_{n^{\prime}}$, $\mathcal{F}_{\left(m^{\prime}, n^{\prime}\right)}$ and $\mathcal{F}_{\left(m^{\prime}, n^{\prime}\right)}^{u^{\prime}}$ are connected to each other keeping the inside $\mathbb{C}^{3}$ untouched (cf. Remark 3.2).

Remark 3.2 (Definitions of $\mathcal{F}_{n}, \mathcal{F}_{(m, n)}, \mathcal{F}_{(m, n)}^{u}$ and elementary transformations).

(1) By definition, $\mathcal{F}_{n}$ is just a $\mathbb{P}^{1}$-bundle structure over $\mathbb{P}^{2}: \mathcal{F}_{n}:=\mathbb{P}\left(\mathcal{O}_{\mathbb{P}^{2}} \oplus \mathcal{O}_{\mathbb{P}^{2}}(n)\right)$. It is easy to construct a sequence of elementary transformations

$$
\chi: \mathbb{P}^{3} /\{\text { pt. }\} \leftarrow \mathcal{F}_{1} / \mathbb{P}^{2}-->\mathcal{F}_{2} / \mathbb{P}^{2}--->\mathcal{F}_{n-1} / \mathbb{P}^{2}-->\mathcal{F}_{n} / \mathbb{P}^{2},
$$

in such a way that $\chi$ has no influence on the inside affine 3 -space $\mathbb{C}^{3}$. In fact, $\mathbb{P}^{3} \leftarrow \mathcal{F}_{1}$ is just the blow-up along the maximal ideal of a point $p$ on the hyperplane at infinity $H_{\infty}$ with the exceptional divisor $E_{1} \cong \mathbb{P}^{2}$ corresponding to the natural surjection $\mathcal{O}_{\mathbb{P}^{2}} \oplus \mathcal{O}_{\mathbb{P}^{2}}(1) \rightarrow \mathcal{O}_{\mathbb{P}^{2}}$. Let $H_{1} \cong \mathbb{F}_{1}$ be the proper transform of $H_{\infty}$ on $\mathcal{F}_{1}$. Let $\operatorname{lm}_{l_{1}}: \mathcal{F}_{1}-\rightarrow \mathcal{F}_{2}$ be the elementary transformation along $l_{1}:=H_{1} \cap E_{1}$, that is, we have $\operatorname{elm}_{l_{1}}=\pi_{1} \circ \rho_{1}^{-1}$, where $\rho_{1}: T_{1} \rightarrow \mathcal{F}_{1}$ is the blow-up along $l_{1}$, and $\pi_{1}: T_{1} \rightarrow \mathcal{F}_{2}$ is the contraction of the proper transform of $H_{1}$ onto a curve. Let $H_{2}$ and $E_{2}$ be the proper transforms on $\mathcal{F}_{2}$ of $\operatorname{Exc}\left(\rho_{1}\right)$ and $E_{1}$, respectively, and put $l_{2}:=H_{2} \cap E_{2}$. Note that $\mathbb{P}^{3} \backslash H_{\infty} \cong \mathcal{F}_{1} \backslash\left(H_{1} \cup E_{1}\right) \cong \mathcal{F}_{2} \backslash\left(H_{2} \cup E_{2}\right) \cong \mathbb{C}^{3}$. In the similar manner, we can construct elementary transformations $\operatorname{elm}_{l_{i}}: \mathcal{F}_{i}-->\mathcal{F}_{i+1}$ inductively, in such a way that they do not bring any change on the inside $\mathbb{C}^{3}$.

(2) Before the definition of $\mathcal{F}_{(m, n)}^{u}$, we need to define $\mathcal{F}_{(m, n)}$ with $n \geqslant m \geqslant 0$, and birational maps among them. By definition, $\mathcal{F}_{(m, n)}$ is a $\mathbb{P}^{2}$-bundle structure over $\mathbb{P}^{1}$ :

$$
\mathcal{F}_{(m, n)}:=\mathbb{P}\left(\mathcal{O}_{\mathbb{P}^{1}} \oplus \mathcal{O}_{\mathbb{P}^{1}}(m) \oplus \mathcal{O}_{\mathbb{P}^{1}}(n)\right),
$$




\section{T. Kishimoto}

and let $B_{(m, n)} \cong \mathbb{F}_{n}$ (respectively $C_{(m, n)} \cong \mathbb{F}_{m}$ ) be a divisor on $\mathcal{F}_{(m, n)}$ corresponding to the surjection

$$
\mathcal{O}_{\mathbb{P}^{1}} \oplus \mathcal{O}_{\mathbb{P}^{1}}(m) \oplus \mathcal{O}_{\mathbb{P}^{1}}(n) \rightarrow \mathcal{O}_{\mathbb{P}^{1}} \oplus \mathcal{O}_{\mathbb{P}^{1}}(n)
$$

(respectively $\mathcal{O}_{\mathbb{P}^{1}} \oplus \mathcal{O}_{\mathbb{P}^{1}}(m) \oplus \mathcal{O}_{\mathbb{P}^{1}}(n) \rightarrow \mathcal{O}_{\mathbb{P}^{1}} \oplus \mathcal{O}_{\mathbb{P}^{1}}(m)$ ). Let $H_{(m, n)}$ be a fiber of a $\mathbb{P}^{2}$-bundle $\mathcal{F}_{(m, n)} / \mathbb{P}^{1}$, and let $l_{(m, n)}:=C_{(m, n)} \cap H_{(m, n)}$ and $l_{(m, n)}^{\prime}:=B_{(m, n)} \cap H_{(m, n)}$. Then we have elementary transformations along $l_{(m, n)}$ and $l_{(m, n)}^{\prime}$, respectively, say

$$
\operatorname{elm}_{l_{(m, n)}}: \mathcal{F}_{(m, n)}--\rightarrow \mathcal{F}_{(m, n+1)} \quad \text { and } \quad \operatorname{elm}_{l_{(m, n)}^{\prime}}: \mathcal{F}_{(m, n)}-->\mathcal{F}_{(m+1, n)}
$$

namely, we have $\operatorname{elm}_{l_{(m, n)}}=\pi_{(m, n)} \circ \rho_{(m, n)}^{-1}$ and $\operatorname{elm}_{l_{(m, n)}^{\prime}}=\pi_{(m, n)}^{\prime} \circ \rho_{(m, n)}^{\prime-1}$, where $\rho_{(m, n)}$ (respectively $\rho_{(m, n)}^{\prime}$ ) is the blow-up along $l_{(m, n)}$ (respectively $l_{(m, n)}^{\prime}$ ), and $\pi_{(m, n)}$ (respectively $\pi_{(m, n)}^{\prime}$ ) is the contraction of the proper transform of $H_{(m, n)}$ onto a smooth point. Let $B_{(m, n+1)}, C_{(m, n+1)}$ and $H_{(m, n+1)}$ (respectively $B_{(m+1, n)}, C_{(m+1, n)}$ and $H_{(m+1, n)}$ ) be proper transforms of $B_{(m, n)}, C_{(m, n)}$ and $\operatorname{Exc}\left(\rho_{(m, n)}^{-1}\right)\left(\right.$ respectively $B_{(m, n)}, C_{(m, n)}$ and $\left.\operatorname{Exc}\left(\rho_{(m, n)}^{\prime-1}\right)\right)$ on $\mathcal{F}_{(m, n+1)}$ (respectively $\left.\mathcal{F}_{(m+1, n)}\right)$. Moreover, we put $l_{(m, n+1)}:=C_{(m, n+1)} \cap H_{(m, n+1)}$ and $l_{(m, n+1)}^{\prime}:=B_{(m, n+1)} \cap H_{(m, n+1)}$ on $\mathcal{F}_{(m, n+1)}$, and $l_{(m+1, n)}:=C_{(m+1, n)} \cap H_{(m+1, n)}$ and $l_{(m+1, n)}^{\prime}:=B_{(m+1, n)} \cap H_{(m+1, n)}$ on $\mathcal{F}_{(m+1, n)}$.

(3) Let $\mathcal{F}_{(m, n)}^{0}:=\mathcal{F}_{(m, n)}$ be as in (2) with $n \geqslant m \geqslant 0$. Then the algebraic 3 -fold $\mathcal{F}_{(m, n)}^{1}$ is obtained from $\mathcal{F}_{(m, n)}^{0}$ via just the blow-up along the curve $B_{(m, n)} \cap C_{(m, n)}$ with the exceptional divisor $D_{(m, n)}^{1} \cong \mathbb{F}_{n-m}$. Let $B_{(m, n)}^{1}$ (respectively $\left.C_{(m, n)}^{1}\right)$ be the proper transform on $\mathcal{F}_{(m, n)}^{1}$ of $B_{(m, n)}$ (respectively $C_{(m, n)}$ ). Let $\operatorname{elm}_{t_{(m, n)}^{1}}: \mathcal{F}_{(m, n)}^{1}-\rightarrow \mathcal{F}_{(m, n)}^{2}$ be the elementary transformation along the curve $t_{(m, n)}^{1}:=C_{(m, n)}^{1} \cap D_{(m, n)}^{1}$, namely, we have

$$
\operatorname{elm}_{t_{(m, n)}^{1}}=\pi_{(m, n)}^{1} \circ \rho_{(m, n)}^{1}{ }^{-1},
$$

where $\rho_{(m, n)}^{1}: U_{(m, n)}^{1} \rightarrow \mathcal{F}_{(m, n)}^{1}$ is the blow-up along $t_{(m, n)}^{1}$, and $\pi_{(m, n)}^{1}: U_{(m, n)}^{1} \rightarrow \mathcal{F}_{(m, n)}^{2}$ is the contraction of the proper transform on $U_{(m, n)}^{1}$ of $C_{(m, n)}^{1}$ onto a curve. Let $C_{(m, n)}^{2}$ (respectively $\left.D_{(m, n)}^{2}\right)$ be the proper transform on $\mathcal{F}_{(m, n)}^{2}$ of $\operatorname{Exc}\left(\rho_{(m, n)}^{1}\right)$ (respectively $\left.D_{(m, n)}^{1}\right)$, and put $t_{(m, n)}^{2}:=$ $C_{(m, n)}^{2} \cap D_{(m, n)}^{2}$. In similar fashion, we can construct birational maps elm $\operatorname{tim}_{(m, n)}^{i}: \mathcal{F}_{(m, n)}^{i}-->\mathcal{F}_{(m, n)}^{i+1}$ along a curve $t_{(m, n)}^{i}:=C_{(m, n)}^{i} \cap D_{(m, n)}^{i}$ inductively, in such a way that they do not bring any change on the inside affine part $\mathbb{C}^{3}$. It is easy to see that $\mathcal{F}_{(m, n)}^{u}$ has a structure of an $\mathbb{F}_{u}$-bundle over $\mathbb{P}^{1}$.

(4) By using elementary transformations in (2) and (3) above, we can construct a sequence of birational maps:

$$
\begin{aligned}
\chi^{\prime}: \mathbb{P}^{3} /\{\text { pt. }\} \leftarrow \mathcal{F}_{(0,1)} / \mathbb{P}^{1}-->\mathcal{F}_{(0,2)} / \mathbb{P}^{1}--->\mathcal{F}_{(0, n)} / \mathbb{P}^{1} \\
-->\mathcal{F}_{(1, n)} / \mathbb{P}^{1}--->\mathcal{F}_{(m, n)} / \mathbb{P}^{1},
\end{aligned}
$$

and

$$
\chi^{\prime \prime}: \mathcal{F}_{(m, n)} / \mathbb{P}^{1} \leftarrow \mathcal{F}_{(m, n)}^{1} / \mathbb{P}^{1}--->\mathcal{F}_{(m, n)}^{u-1} / \mathbb{P}^{1}-->\mathcal{F}_{(m, n)}^{u} / \mathbb{P}^{1}
$$

in such a way that the composite $\chi:=\chi^{\prime \prime} \circ \chi^{\prime}$ does not have any effect on the inside affine 3 -space $\mathbb{C}^{3}$.

Remark 3.3. The 3 -folds which we can choose as $V$ in Theorem 3.1 depend on the form of an automorphism $\alpha \in J_{3}$ of de Jonquière type. If $\alpha \in J_{3}$ is of the form:

$$
\alpha:\left\{\begin{array}{l}
x \mapsto a x+g(y, z), \\
y \mapsto b y+b^{\prime} z+b^{\prime \prime}, \\
z \mapsto c z+c^{\prime},
\end{array}\right.
$$

with $a b c \neq 0$, then we can choose $V \cong \mathcal{F}_{n}$ in such a way that $\operatorname{deg} g(y, z) \leqslant n$. On the other hand, 
assume that $\alpha \in J_{3}$ is of the form:

$$
\alpha:\left\{\begin{array}{l}
x \mapsto a x+g(y, z), \\
y \mapsto b y+h(z), \\
z \mapsto c z+c^{\prime},
\end{array}\right.
$$

with $a b c \neq 0, h(z) \in \mathbb{C}[z] \backslash \mathbb{C}, d_{1}:=\operatorname{deg} h(z)>0, d_{2}:=\operatorname{deg}_{y} g(y, z)$. Let $\delta:=\operatorname{wt} g(y, z)$ be the weighted degree of $g$ with respect to wt $(y)=d_{1}$ and wt $(z)=1$. Then by choosing $n$ and $m$ in such a way that $n \geqslant \max \left\{d_{1}, d_{2}, \delta\right\}$ and $m=n-d_{1}$, we can take $V \cong \mathcal{F}_{(m, n)}^{n}$ (see [Fre95] for a more detailed explanation).

In order to prove Theorem 1.1, we prepare the following lemma.

Lemma 3.2. Let $\alpha \in J_{3}$ be an automorphism of de Jonquière type, and let $\Phi_{\alpha}: X=\mathbb{P}^{3}-->X^{\prime}=$ $\mathbb{P}^{3}$ be the Cremona transformation induced by $\alpha$ in a natural way. Let $\nu$ be a discrete valuation whose center $\operatorname{Center}_{X}(\nu)$ on $X$ is either a point or a line on the hyperplane at infinity $H_{\infty}=X \backslash \mathbb{C}^{3}$, or $H_{\infty}$ itself. Then the center $\operatorname{Center}_{X^{\prime}}(\nu)$ of $\nu$ on $X^{\prime}$ is either a point or a line on $H_{\infty}^{\prime}:=X^{\prime} \backslash \alpha\left(\mathbb{C}^{3}\right)$, or $H_{\infty}^{\prime}$ itself.

Proof. In the case where $\alpha$ is linear, the assertion is clear, so we may assume that $\alpha \in J_{3} \backslash A_{3}$ in what follows. By Theorem 3.1, there exist an algebraic threefold $V$, and a birational map $\chi: \mathbb{P}^{3}-->V$, which is isomorphic when restricted onto the inside affine 3 -space $\mathbb{C}^{3}$, such that an automorphism $\alpha$ can be extended to an automorphism $\varphi_{\alpha}: V \stackrel{\sim}{\rightarrow} V$ which fits into the commutative diagram in Remark 3.1; in particular, we have $\Phi_{\alpha}=\chi^{-1} \circ \varphi_{\alpha} \circ \chi$. More precisely, $\chi$ is expressed as a composite of elementary transformations as mentioned in Remark 3.2(1), (2) and (3). Let $h: U \rightarrow V$ be a composite of blow-ups of smooth centers which extracts all the discrete valuations influenced by the process $\chi$, such that $g:=\chi^{-1} \circ h: U \rightarrow \mathbb{P}^{3}$ gives rise to a morphism and $\varphi_{\alpha}$ is extended to an automorphism on $U$, say $\phi_{\alpha}: U \stackrel{\sim}{\rightarrow} U$. In other words, $\mathbb{P}^{3} \stackrel{g}{\leftarrow} U \stackrel{g \circ \phi_{\alpha}}{\rightarrow} \mathbb{P}^{3}$ is a minimal common resolution of $\Phi_{\alpha}$. More precisely, let $\left\{E_{j}\right\}_{j=1}^{r}$ exhaust the $g$-exceptional divisors and let $E_{0}$ be the proper transform on $U$ of $H_{\infty}$. Then $\phi_{\alpha}$ also induces a suitable permutation of these divisors $\phi_{\alpha}:\left\{E_{0}, E_{1}, \cdots, E_{r}\right\} \stackrel{\sim}{\rightarrow}\left\{E_{0}, E_{1}, \cdots, E_{r}\right\}$ and $g \circ \phi_{\alpha}$ contracts $\left\{E_{j}\right\}_{j=0}^{r}$ except for exactly one component distinct from $E_{0}$, which becomes $H_{\infty}^{\prime}$ on $X^{\prime}$. We then note the following.

Claim. Center $_{X}\left(E_{j}\right)=g\left(E_{j}\right)$ is either a point or a line on $H_{\infty}(1 \leqslant j \leqslant r)$.

Proof of claim. Since $\alpha$ is of de Jonquière type, it is not difficult to see the assertion. Indeed, let:

$$
\alpha:\left\{\begin{array}{l}
x \mapsto p(x, y, z)=a x+g(y, z) \\
y \mapsto q(x, y, z)=b y+h(z) \\
z \mapsto r(x, y, z)=c z+c^{\prime},
\end{array}\right.
$$

where $a b c \neq 0$. We consider the case where $d:=\max \{\operatorname{deg}(p), \operatorname{deg}(q), \operatorname{deg}(r)\}=\operatorname{deg}(p)$. The other case also can be ascertained by the similar argument. Let $P(x, y, z, t), Q(x, y, z, t), R(x, y, z, t)$ be the corresponding homogeneous polynomials, where $H_{\infty}$ is defined by $t=0$. Then the base locus of the mobile linear system $\Phi_{\alpha_{*}}^{-1}\left|\mathcal{O}_{\mathbb{P}^{3}}(1)\right|$ is contained in the plane curve $B:=(P(x, y, z, 0)=0) \subset$ $H_{\infty} \cong \mathbb{P}^{2}[x: y: z]$. Note that $P(x, y, z, 0)$ coincides with the highest degree part of $p(x, y, z)$, so that it coincides with the homogeneous part $g_{d}(y, z)$ of degree $d$ of $g(y, z)$. Hence $B$ is composed of several lines on $H_{\infty}$ passing through [1:0:0]. Since the set of points on $X$ where $\Phi_{\alpha}$ is not indeterminate is contained in $B$, the assertion holds true by construction.

We shall prove the assertion of the Lemma. Let $\nu$ be a discrete valuation whose center $\operatorname{Center}_{X}(\nu)$ on $X$ is either a point, or a line in $H_{\infty}$, or $H_{\infty}$ itself. We shall divide into the three cases. 


\section{T. Кіsнiмото}

Case 1. If $\operatorname{Center}_{X}(\nu)$ coincides with $H_{\infty}$, then its center on $U$ is $E_{0}$, and it is contracted via the process $g \circ \phi_{\alpha}$. By the symmetry between $g$ and $g \circ \phi_{\alpha}$ (up to $\phi_{\alpha}$ ), it follows that its center Center $_{X^{\prime}}(\nu)=\left(g \circ \phi_{\alpha}\right)\left(E_{0}\right)$ on $X^{\prime}$ is either a point or a line in $H_{\infty}^{\prime}$ in consideration of the Claim.

Case 2. Let us consider the case where $\operatorname{Center}_{U}(\nu)$ coincides with $E_{j}$ for some $1 \leqslant j \leqslant r$. If $E_{j}$ is contracted via $g \circ \phi_{\alpha}$, then the assertion holds true by the same argument as in Case 1 . On the other hand, if $E_{j}$ is not contracted by $g \circ \phi_{\alpha}$, this implies that the center $\operatorname{Center}_{X^{\prime}}(\nu)=\left(g \circ \phi_{\alpha}\right)\left(E_{j}\right)$ coincides with $H_{\infty}^{\prime}$.

Case 3. Finally, if $\nu$ does not correspond to Case 1 or Case 2, then $\operatorname{Center}_{U}(\nu)$ is a point or a curve in $E_{0}$. In either case, by noting that $E_{0}$ is contracted via $g \circ \phi_{\alpha}$, it follows that $\operatorname{Center}_{X^{\prime}}(\nu)=$ $\left(g \circ \phi_{\alpha}\right)\left(\right.$ Center $\left._{U}(\nu)\right)$ is either a point or a line by virtue of the Claim and by the symmetry between the process of $g$ and $g \circ \phi_{\alpha}$.

Thus we complete the proof of the Lemma.

\section{Proof of Theorem 1.1}

We shall use the same notation as before Theorem 3.1. Our proof consists of three steps.

Step 1. First of all, we write $\theta$ as a composite of affine and de Jonquière transformation as in $(*)$ to obtain the expression $(* * *)$ after performing Sarkisov factorization to each $\Phi_{\sigma_{i}}$ as in $(*)_{i}$. On the other hand, let

$$
\Phi_{\theta}=\chi_{s}^{\prime} \circ \cdots \circ \chi_{1}^{\prime}
$$

be a Sarkisov factorization of $\Phi_{\theta}$. Then we make the following claim.

Claim 1. The (strong) maximal singularity of $\chi_{1}^{\prime}$ (cf. Definition 2.2) is extracted in the procedure $\chi_{k}^{(j)}$ in (***) for some $1 \leqslant j \leqslant r$ and $1 \leqslant k \leqslant r_{k}$.

Proof of Claim 1. Assume to the contrary that the strong maximal singularity of $\chi_{1}^{\prime}$, say $\nu$, does not appear in any of the $\chi_{k}^{(j)}$. Then, the birational map $\Phi_{\theta}$ is isomorphic along $\nu$. In particular, this implies that $K_{\mathbb{P}^{3}}+(1 / \mu) \mathcal{H}$ is canonical along $\nu$ for any $\mathcal{H}:=\Phi_{\theta_{*}}^{-1}\left|\mathcal{O}_{\mathbb{P}^{3}}(k)\right|$, where $\mu$ is a quasi-effective threshold of the pair $\left(\mathbb{P}^{3}, \mathcal{H}\right)$ (see $\S 2$ ). This is a contradiction.

Step 2. Let $\nu$ be the strong maximal singularity of $\chi_{1}^{\prime}$. We need to see that the center $\operatorname{Center}_{\mathbb{P}^{3}}(\nu)$ of $\nu$ on the starting projective 3 -space $\mathbb{P}^{3}$ is either a point or a line on the hyperplane at infinity $H_{\infty}$. By Claim 1, $\nu$ appears in a suitable $\chi_{k}^{(j)}$. This elementary link $\chi_{k}^{(j)}$ itself appears in the Sarkisov factorization of $\Phi_{\sigma_{j}}$ :

$$
\begin{aligned}
\Phi_{\sigma_{j}}=\chi_{r_{j}}^{(j)} \circ \cdots \circ \chi_{1}^{(j)}: X_{0}^{(j)} / S_{0}^{(j)}:=\mathbb{P}^{3} /\{\text { pt. }\} & ->X_{1}^{(j)} / S_{1}^{(j)} \\
& ---->X_{r_{j}}^{(j)} / S_{r_{j}}^{(j)}:=\mathbb{P}^{3} /\{\text { pt. }\} .
\end{aligned}
$$

Since $\sigma_{j}$ is of de Jonquière type, there exist an algebraic 3-fold $V$, a birational map $\chi: \mathbb{P}^{3}-->V$ and an automorphism $\varphi: V \stackrel{\sim}{\rightarrow} V$ such that $\Phi_{\sigma_{j}}=\chi^{-1} \circ \varphi \circ \chi$ by virtue of Theorem 3.1. Moreover, $\chi$ is decomposed into elementary transformations as mentioned in Remark 3.2. Then, as in Claim 1, we make the following claim.

Claim 2. The singularly $\nu$ is extracted in some elementary transformation appearing in $\chi^{-1} \circ \varphi \circ \chi$.

Proof of Claim 2. Otherwise, $\Phi_{\sigma_{j}}$ is isomorphic along $\nu$, in particular, $X_{k-1}^{(j)} / S_{k-1}^{(j)}-->X_{r_{j}}^{(j)} / S_{r_{j}}^{(j)}$ $=\mathbb{P}^{3} /\{$ pt. $\}$ is isomorphic along $\nu$. This implies that $K_{X_{k-1}^{(j)}}+(1 / \mu) \mathcal{H}$ is canonical along $\nu$ for any mobile linear system $\mathcal{H}$, which is a proper transform on $X_{k-1}^{(j)}$ of a very ample complete linear system on $X_{r_{j}}^{(j)}$, with $\mu$ the quasi-effective threshold of $\left(X_{k-1}^{(j)}, \mathcal{H}\right)$ (see $\left.\S 2\right)$. This is a contradiction. 
Step 3. By Claim 2, the discrete valuation $\nu$ in question appears in a suitable elementary transformation in $\chi^{-1} \circ \varphi \circ \chi=\Phi_{\sigma_{j}}: X=\mathbb{P}^{3}-->X^{\prime}=\mathbb{P}^{3}$. In particular, $\operatorname{Center}_{X}(\nu)$ is either a point or a line, or the hyperplane at infinity itself, in consideration of the procedure of elementary transformations. Then repeated application of Lemma 3.2 for $\sigma_{j-1}^{-1}, \ldots, \sigma_{1}^{-1}$ implies that the center $\operatorname{Center}_{\mathbb{P}^{3}}(\nu)$ on the starting $\mathbb{P}^{3}$ is either a point or a line in the hyperplane at infinity $H_{\infty}$, or $H_{\infty}$ itself. Note that the case where $\operatorname{Center}_{\mathbb{P}^{3}}(\nu)$ coincides with $H_{\infty}$ is obviously not possible by the mechanism of the Sarkisov Program.

Thus we complete the proof of Theorem 1.1 by using Proposition 3.1.

Remark 3.4. Although Theorem 1.1 and the main results due to Shestakov and Umirbaev in [SU04b, Theorem 2, Corollary 8] give us certain necessary conditions for a given automorphism $\theta \in G_{3}$ on $\mathbb{C}^{3}$ to be tame, and are enough to prove the non-tameness of the Nagata automorphism (cf. Theorem 1.2; see also [SU04b, Corollary 9]) and many other automorphisms (see $\S 4$, Example 4.1), they are not sufficient conditions. In fact, there exists an example of $\theta \in G_{3}$ which is not tame, nevertheless Theorem 1.1 and [SU04b, Theorem 2, Corollary 8] cannot recognize that $\theta$ is not tame (cf. $\S 4$, Example 4.2).

\section{A new proof of the non-tameness of the Nagata automorphism}

In this section, we shall give a new proof for the result on the non-tameness of the Nagata automorphism $\sigma$ due to Shestakov and Umirbaev (cf. [SU04a, SU04b]), as an application of Theorem 1.1. Let $\Phi_{\sigma}: \mathbb{P}^{3}-->\mathbb{P}^{3}$ be the Cremona transformation induced by $\sigma$ in a natural manner, namely, it is defined as follows (see $\S 1$ ):

$$
\Phi_{\sigma}:\left\{\begin{array}{l}
x \mapsto F:=x t^{4}-2 y\left(x z+y^{2}\right) t^{2}-z\left(x z+y^{2}\right)^{2}, \\
y \mapsto G:=\left(y t^{2}+z\left(x z+y^{2}\right)\right) t^{2}, \\
z \mapsto H:=z t^{4} \\
t \mapsto I:=t^{5},
\end{array}\right.
$$

where $t=0$ defines the hyperplane at infinity $H_{\infty}$ with respect to the canonical open immersion $\mathbb{C}^{3}{ }_{(x, y, z)} \hookrightarrow \mathbb{P}^{3}{ }_{[x: y: z: t]}$.

Remark 4.1. By virtue of Theorem 1.1, once we have succeeded in the explicit Sarkisov factorization of $\Phi_{\sigma}$ as in [Kis05], it is easy to see that $\sigma$ is not tame. Indeed, we obtain the Sarkisov factorization of $\Phi_{\sigma}$ into eight elementary links, say

$$
\Phi_{\sigma}=\chi_{8} \circ \cdots \circ \chi_{1}
$$

such that the first elementary link $\chi_{1}$ starts with the blow-up along a smooth conic on $H_{\infty}$ (cf. [Kis05]). As a consequence, it follows that the Nagata automorphism $\sigma$ is not tame by Theorem 1.1. However, for a given automorphism $\tau \in G_{3}$ on $\mathbb{C}^{3}$, it is usually difficult to perform the explicit Sarkisov factorization of the Cremona transformation $\Phi_{\tau}$ induced by $\tau$. For the further application of Theorem 1.1, it is useful to develop a technique to look into (non-)tameness that does not depend on the explicit Sarkisov factorizations. Theorem 1.1 indicates that the essential idea often lies in the maximal center of the first elementary link in a suitable Sarkisov factorization.

We shall, now, give a new proof of the non-tameness of the Nagata automorphism $\sigma$, which relies only on a maximal center of the first elementary link and does not depend on the explicit Sarkisov factorization itself. In our strategy to determine the maximal center, we make use of the technique in [Cor00, Corollary 3.4] due to Corti, which is one of the consequences of inversion of adjunction [Kol92, Chapters 16 and 17]. 


\section{T. Кіsнiмото}

\section{Proof of Theorem 1.2}

Our proof consists of three steps. Let $\sigma$ be the Nagata automorphism, and let $\Phi_{\sigma}: \mathbb{P}^{3}-->\mathbb{P}^{3}$ be the Cremona transformation induced by $\sigma$ (see the beginning of this section).

Step 1. At first, we shall investigate a factorization of $\Phi_{\sigma}$ into elementary links according to the Sarkisov program. For this purpose, we consider a very ample complete linear system $\left|\mathcal{O}_{\mathbb{P}^{3}}(d)\right|$ on the target $\mathbb{P}^{3}$ and its proper transform $\mathcal{H}:=\Phi_{\sigma *}{ }^{-1}\left|\mathcal{O}_{\mathbb{P}^{3}}(d)\right|$ by $\Phi_{\sigma}$. By the equation of $\Phi_{\sigma}$, the linear system $\mathcal{H}$ is described as follows:

$$
\mathcal{H}=\left\langle F^{\alpha} G^{\beta} H^{\gamma} I^{\delta} \mid \alpha+\beta+\gamma+\delta=d ; \alpha, \beta, \gamma, \delta \geqslant 0\right\rangle,
$$

which means that $\mathcal{H}$ is spanned by hypersurfaces in $\mathbb{P}^{3}$ defined by $F^{\alpha} G^{\beta} H^{\gamma} I^{\delta}=0(\alpha+\beta+\gamma+\delta=d)$. It is easy to see that the base locus Bs $\mathcal{H}$ coincides set-theoretically with $C_{0} \cup L_{0}$, where $C_{0}:=(t=$ $\left.x z+y^{2}=0\right)$ and $L_{0}:=(t=z=0)$. By the mechanism of the Sarkisov program (cf. [Cor95] or $\S 2$ ), we need to determine the Sarkisov degree $(\mu, c, e)$ of the pair $\left(\mathbb{P}^{3}, \mathcal{H}\right)$ (cf. Definition 2.1), and look for a maximal center and a maximal divisorial blow-up (cf. Definition 2.2) in order to construct the first elementary link. (Note that a maximal center is contained in Bs $\mathcal{H}$.) Since $\mathcal{H} \subset\left|\mathcal{O}_{\mathbb{P}^{3}}(5 d)\right|$, we have $\mu=5 d / 4$. Let $S \in \mathcal{H}$ be a general member. Then it follows that mult $C_{0} S=2 d$ and mult $_{L_{0}} S=d$, hence we can easily see that the canonical threshold $c$ of $\left(\mathbb{P}^{3}, \mathcal{H}\right)$ is less than or equal to $1 / 2 d$, and the line $L_{0}$ cannot be a maximal center.

Step 2. In fact, we have the following claim concerning the possibility of a maximal center. This is the crucial part in our proof.

Claim. The conic $C_{0}$ is a unique maximal center and $c=1 / 2 d$. In other words, any point and any curve different from $C_{0}$ cannot be a maximal center.

Proof of Claim. Note that a maximal center is contained in Bs $\mathcal{H}=C_{0} \cup L_{0}$ (cf. Remark 2.1). Since we have already excluded $L_{0}$ as a maximal center, we have to verify that any discrete valuation $E$ of $\mathbb{C}\left(\mathbb{P}^{3}\right)$ exceptional over $\mathbb{P}^{3}$ with $\operatorname{Center}_{\mathbb{P}^{3}}(E)=\{$ pt. $\}$ is canonical with respect to $K_{\mathbb{P}^{3}}+(1 / 2 d+\epsilon) \mathcal{H}$ for a sufficiently small $0<\epsilon \ll 1$. Assume to the contrary that a point $p$ contained in $C_{0} \cup L_{0}$ is a maximal center, i.e. $K_{\mathbb{P} 3}+(1 / 2 d+\epsilon) \mathcal{H}$ is not canonical at $p$ for $0<\epsilon \ll 1$. Let $\mathcal{H}_{0}$ be a mobile sublinear system of $\mathcal{H}$ spanned by $S$ and $5 d H_{\infty}$, where $S$ is a general member of $\mathcal{H}$. Then $K_{\mathbb{P}^{3}}+(1 / 2 d+\epsilon) \mathcal{H}_{0}$ is not canonical at $p$. Thus, by virtue of [Cor00, Corollary 3.4], we have the following inequality concerning multiplicity:

$$
\operatorname{mult}_{p} S_{1} \cdot S_{2}>4\left(\frac{2 d}{1+2 d \epsilon}\right)^{2}
$$

where $S_{1} \cdot S_{2}$ stands for a scheme-theoretic intersection of two general members $S_{1}, S_{2}$ of $\mathcal{H}_{0}$. On the other hand, it is not difficult to see that $S_{1} \cdot S_{2}=5 d^{2}\left(2 C_{0}+L_{0}\right)$. Hence we have $\operatorname{mult}_{p} S_{1} \cdot S_{2} \leqslant 15 d^{2}$ (the equality mult $S_{1} \cdot S_{2}=15 d^{2}$ is attained only when $p=C_{0} \cap L_{0}$ ). Thus we have a contradiction by choosing $\epsilon$ sufficiently small. Thus we see that $C_{0}$ is a unique maximal center. The remaining assertion $c=1 / 2 d$ is then easy to see.

Step 3. By the previous claim, we know that, for a Sarkisov factorization of $\Phi_{\sigma}$, the first elementary link is unique and it starts with the blow-up along the conic $C_{0}$. On the other hand, provided that the Nagata automorphism $\sigma$ is tame, the maximal center of the first elementary link appearing in any Sarkisov factorization of $\Phi_{\sigma}$ is either a point or a line (Theorem 1.1). This is a contradiction. Thus it follows that $\sigma$ is not tame as desired.

This completes the proof of Theorem 1.2. 


\section{Proof of NON-TAMENESS OF THE NAGATA AUtOMORPhisM}

Example 4.1 (cf. Bass [Bas84]). Let $\Delta$ be the locally nilpotent derivation on $\mathbb{C}[x, y, z]$ of the form $\Delta=z \partial_{y}-2 y \partial_{x}$, and let $F:=x z+y^{2}$. It is easy to see that $D:=F \Delta$ is also locally nilpotent, hence it yields an algebraic $\mathbb{C}_{+}$-action on $\mathbb{C}^{3}$, in other words, a $\mathbb{C}$-algebra homomorphism (cf. [Miy78]):

$$
\varphi_{D}: \mathbb{C}[x, y, z] \rightarrow \mathbb{C}[x, y, z][t], \quad h \mapsto \sum_{k \geqslant 0} \frac{D^{k}(h)}{k !} t^{k},
$$

where $t$ is a coordinate on $\mathbb{C}_{+}$. For each $t \in \mathbb{C}_{+}$fixed, $\varphi_{D}$ gives rise to an exponential automorphism:

$$
\sigma_{t}:=\exp (t D):(x, y, z) \mapsto\left(x-2 t y F-t^{2} z F^{2}, y+t z F, z\right) .
$$

(Note that, in the case of $t=1, \sigma_{1}$ is just the Nagata automorphism.) We can show that $\sigma_{t}$ is not tame by the same argument as in the proof of Theorem 1.2 for $t \neq 0$.

Example 4.2. Both Theorem 1.1 and the main results [SU04b, Theorem 2, Corollary 8] do not give us sufficient conditions for the tameness. For example, let $\sigma$ be the Nagata automorphism as usual, and let $\tau \in J_{3}$ be defined as follows:

$$
\tau:\left\{\begin{array}{l}
x \mapsto x+y^{2}+z, \\
y \mapsto y+z^{2} \\
z \mapsto z
\end{array}\right.
$$

Then the composite $\theta:=\tau \circ \sigma$ is defined as:

$$
\theta:\left\{\begin{array}{l}
x \mapsto-z^{9}+\text { (lower degree terms), } \\
y \mapsto z^{5}+y^{2} z^{2}+2 y z^{3}+x z^{2}+y^{2} z+z^{3}+z^{2}+y, \\
z \mapsto z
\end{array}\right.
$$

Note that since $\sigma$ is not tame, $\theta$ is also obviously not tame. However, we have the following.

(a) Composite $\theta$ admits an elementary reduction in the sense of [SU04b, p. 204]. Therefore, we cannot conclude that $\theta$ is not tame from [SU04b, Theorem 2, Corollary 8].

(b) The first elementary link appearing in the Sarkisov factorization of $\Phi_{\theta}$ starts with a divisorial blow-up with a maximal center contained in the line $(t=z=0)$ on the hyperplane at infinity $H_{\infty}=(t=0)$. Hence, we cannot deduce that $\theta$ is not tame from Theorem 1.1.

As the above mentioned example indicates, for a non-tame automorphism on $\mathbb{C}^{3}$ which is obtained as a composite of non-tame and tame automorphisms, Theorem 1.1 is not effective.

Remark 4.2. In consideration of Example 4.2, it is helpful to define a certain class of non-tame automorphisms on $\mathbb{C}^{3}$. For $\theta \in G_{3}$, we put $\operatorname{deg} \theta:=\operatorname{deg} \theta(x)+\operatorname{deg} \theta(y)+\operatorname{deg} \theta(z)$. An automorphism $\theta$ is said to be non-essential if at least one of $\operatorname{deg}(\tau \circ \theta)$ and $\operatorname{deg}(\theta \circ \tau)$ is strictly smaller than $\operatorname{deg} \theta$ by using a suitable tame automorphism $\tau \in T_{3}$. Note that any automorphism in $T_{3} \backslash A_{3}$ is non-essential. If $\theta$ is not non-essential, then we call it essential. Furthermore, if $\theta$ is non-tame and essential, then $\theta$ is said to be essentially non-tame. It is important to classify essentially non-tame automorphisms in order to investigate the structure of $G_{3}$. We can construct some essentially non-tame ones on $\mathbb{C}^{3}$ with reference to the explicit factorization of $\Phi_{\sigma}$ as in [Kis05]. Although we can construct them geometrically, the weak point of our approach lies in the difficulty to write down the equations of such geometrically constructed automorphisms concretely.

For the further investigation of essentially non-tame automorphisms on $\mathbb{C}^{3}$ and the group $G_{3}$, it is important to consider the following problem.

Problem 1. Is there an essentially non-tame automorphism $\theta$ on the affine 3 -space $\mathbb{C}^{3}$ such that the Sarkisov factorization of the Cremona transformation $\Phi_{\theta}$ on $\mathbb{P}^{3}$ induced by $\theta$ starts with a (weighted) blow-up at a point on the hyperplane at infinity? 


\section{T. Кіsнiмото}

To our knowledge, we do not know such an example $\theta \in G_{3}$ satisfying the property in Problem 1 . Once we know that the first elementary link appearing in the Sarkisov factorization of $\Phi_{\theta}$ has a point, say $p$, as a maximal center, it starts with a weighted blow-up of weights wt $(u, v, w)=(1, a, b)$, where $(u, v, w)$ are suitable local coordinates at $p$, and $a, b$ are co-prime positive integers by virtue of the result due to Kawakita [Ka01]. However, as mentioned in [KSC04, pp. 133], the main practical difficulty lies in the fact that $(u, v, w)$ are not necessarily linear with respect to the global coordinates. In consideration of this fact, it is also important to investigate the following problem.

Problem 2. Classify the elementary links $\chi_{1}: \mathbb{P}^{3}--\rightarrow X_{1} / B_{1}$ which start with a weighted blow-up at a point on the hyperplane at infinity.

\section{ACKNOWLEDGEMENTS}

The author would like to express his gratitude to Professors Masayoshi Miyanishi, Mikhail Zaidenberg and Peter Russell for pointing out crucial gaps in the earlier version and for giving him valuable, constructive advice and warm encouragement during the preparation of the present paper. He would like to express his thanks to Professor Tomoaki Ohta for helpful advice to rewrite some inaccurate descriptions. He would also like to thank Professor Hideo Kojima for useful comments and discussions. Also, he would like to express his gratitude to the referee for useful suggestions to improve the description of the paper. Most of the paper was written during the author's stay at Institut Fourier (IF). He would like to express his thanks to IF for their warm hospitality and splendid research environment. The author is supported by JSPS Postdoctoral Fellowships for Research Abroad.

\section{REFERENCES}

AM75 S. S. Abhyankar and T. T. Moh, Embeddings of the line in the plane, J. reine angew. Math. 276 (1975), 148-166.

Bas84 H. Bass, A non-triangular action of $\mathbb{G}_{a}$ on $\mathbb{A}^{3}$, J. Pure Appl. Algebra 33 (1984), 1-5.

Cor95 A. Corti, Factoring birational maps of 3-folds after Sarkisov, J. Algebraic Geom. 4 (1995), $223-254$.

Cor00 A. Corti, Singularities of linear systems and 3-fold birational geometry, in Explicit birational geometry of 3-folds, London Mathematical Society Lecture Note Series, vol. 281 (Cambridge University Press, Cambridge, 2000), 259-312.

CM04 A. Corti and M. Mella, Birational geometry of terminal quartic 3-folds. I, Amer. J. Math. 126 (2004), 739-761.

CPR00 A. Corti, A. Pukhlikov and M. Reid, Fano 3-folds hypersurfaces, in Explicit birational geometry of 3-folds, London Mathematical Society Lecture Note Series, vol. 281 (Cambridge University Press, Cambridge, 2000), 175-258.

Fre95 G. Freudenburg, Three-dimensional analogues of the Nagata surfaces, Comm. Algebra 23 (1995), 139-164.

Ka01 M. Kawakita, Divisorial contractions in dimension three which contract divisors to smooth points, Invent. Math. 145 (2001), 105-119.

Ka02 M. Kawakita, Divisorial contractions in dimension three which contract divisors to compound $A_{1}$ points, Compositio Math. 133 (2002), 95-116.

Ka05 M. Kawakita, Three-fold divisorial contractions to singularities of higher indices, Duke Math. J. 130 (2005), 57-126.

Kaw96 Y. Kawamata, Divisorial contractions to 3-dimensional terminal quotient singularities, in Higherdimensional complex varieties (de Gruyter, Berlin, 1996), 241-246. 


\section{Proof of NON-TAMENESS OF THE NAGATA AUTOMORPHISM}

KMM87 Y. Kawamata, K. Matsuda and K. Matsuki, Introduction to the minimal model problem, in Algebraic geometry, Sendai, 1985, Advanced Studies in Pure Mathematics, vol. 10 (North Holland, Amsterdam, 1987), 283-360.

Kis05 T. Kishimoto, The explicit factorization of the Cremona transformation which is an extension of the Nagata automorphisms into elementary links, Math. Nachr. 278 (2005), 833-843.

Kol92 J. Kollár et al., Flips and abundance for algebraic threefolds, in Papers from the second summer seminar on algebraic geometry, University of Utah, August 1991, Astérisque 211 (1992).

KSC04 J. Kollár, K. Smith and A. Corti, Rational and nearly rational varieties, Cambridge Studies in Advanced Mathematics, vol. 92 (Cambridge University Press, Cambridge, 2004).

Mat01 K. Matsuki, Introduction to the Mori program, Universitext (Springer, Berlin, 2001).

Mel04 M. Mella, Birational geometry of quartic 3-folds. II. The importance of being $\mathbb{Q}$-factorial, Math. Ann. 330 (2004), 107-126.

Miy78 M. Miyanishi, Lectures on curves on rational and unirational surfaces (Tata Institute of Fundamental Research, Bombay, 1978).

Miy01 M. Miyanishi, Open algebraic surfaces, CRM Monograph Series, vol. 12 (American Mathematical Society, Providence, RI, 2001).

Mor82 S. Mori, Threefolds whose canonical bundles are not numerically effective, Ann. of Math. (2) 116 (1982), 133-176.

Nag72 M. Nagata, On automorphism group of $k[x, y]$, Lectures in Mathematics, Department of Mathematics, Kyoto University, vol. 5 (Kinokuniya Book-Store, Tokyo, 1972).

Nag78 M. Nagata, Polynomial rings and affine spaces, Regional Conference Series in Mathematics, vol. 37 (American Mathematical Society, Providence, RI, 1978).

SU04a I. P. Shestakov and U. U. Umirbaev, Poisson brackets and two-generated subalgebras of rings of polynomials, J. Amer. Math. Soc. 17 (2004), 181-196.

SU04b I. P. Shestakov and U. U. Umirbaev, The tame and the wild automorphisms of polynomial rings in three variables, J. Amer. Math. Soc. 17 (2004), 197-227.

Tzi03 N. Tziolas, Terminal 3-fold divisorial contractions of a surface to a curve. I, Compositio Math. 139 (2003), 239-261.

Takashi Kishimoto tkishimo@rimath.saitama-u.ac.jp

Department of Mathematics, Faculty of Science, Saitama University, Saitama 338-8570, Japan 